\title{
Application of Various Normalization Methods for Microscale Analysis of Tissues Using Direct Analyte Probed Nanoextraction
}

\author{
Janella de Jesus, ${ }^{*},+\neq$ Josephine Bunch, ${ }^{*}$ Guido Verbeck, ${ }^{\S}$ Roger P. Webb, " Catia Costa," \\ Richard J. A. Goodwin, ${ }^{\perp}$ and Melanie J. Bailey* ${ }^{\dagger} \dagger$ \\ ${ }^{\dagger}$ Department of Chemistry, University of Surrey, Guildford, Surrey GU2 7XH, United Kingdom \\ ${ }^{*}$ The National Physical Laboratory, Teddington, Middlesex TW11 OLW, United Kingdom \\ ${ }^{\S}$ University of North Texas, Denton, Texas 76203, United States \\ "University of Surrey Ion Beam Centre, Guildford, Surrey GU2 7XH, United Kingdom \\ ${ }^{\perp}$ Pathology Sciences, Drug Safety \& Metabolism, IMED Biotech Unit, AstraZeneca, Cambridge, United Kingdom
}

Supporting Information

ABSTRACT: Direct analyte probed nanoextraction (DAPNe) is a method of extracting material from a microscale region of a sample and provides the opportunity for detailed mass spectrometry analysis of extracted analytes from a small area. The technique has been shown to provide enhanced sensitivity compared with bulk analysis by selectively removing analytes from their matrix and has been applied for selective analysis of single cells and even single organelles. However, the quantitative capabilities of the technique are yet to be fully evaluated. In this study, various normalization techniques were investigated in order to improve the quantitative capabilities of the technique. Two methods of internal standard incorporation were applied to test substrates, which were designed to replicate biological sample matrices. Additionally, normalization to the extraction spot area and matrix compounds were investigated for suitability in situations when an internal standard is not available. The variability observed can be significantly reduced by using a sprayed internal standard and, in some cases, by normalizing to the extracted area.

$\mathrm{D}$ irect analyte probed nanoextraction (DAPNe) is a microscale sample manipulation and extraction technique developed by the group of Verbeck. ${ }^{1}$ The technique is suited to the analysis of small sample volumes and has been demonstrated to be applicable to the extraction and analysis of single cells ${ }^{2}$ and their organelles, ${ }^{3}$ as well as extracting inks and drug residues from different surfaces. ${ }^{4-7}$ The technique is similar in concept to liquid extraction surface analysis (LESA), which is now available as a commercial tool. ${ }^{8,9}$ The major difference in methodology is the smaller sampling area afforded by DAPNe, with a spot diameter of $6 \mu \mathrm{m}$ having been recorded in previous work. ${ }^{7}$ This is compared to a typical LESA spot diameter of around $500 \mu \mathrm{m}$. $^{10}$

A simple schematic of DAPNe is shown in Figure 1. The DAPNe workstation consists of nanomanipulators, which control the movement of a capillary tip in the $x, y$, and $z$ dimensions. The workstation also includes a microinjector, which controls the pressure and time at which an extraction solvent is pushed onto the region of interest and (together with extracted analytes) reaspirated back into the capillary tip. In $\mathrm{DAPNe}$, the sample is collected in a nanospray capillary, giving the opportunity for analysis using mass spectrometry. This affords the opportunity for detailed molecular information from a localized region of the sample to be obtained. For

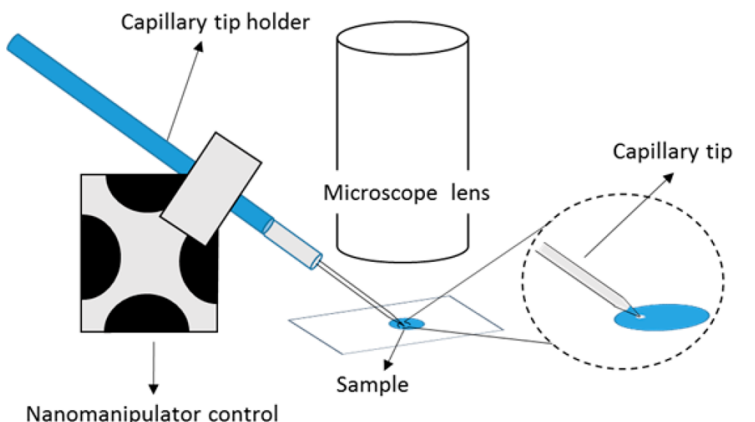

Figure 1. Simple schematic of direct analyte probed nanoextraction (DAPNe).

example, the technique has been shown to have attogram sensitivity to contaminants on surfaces. ${ }^{11}$

While imaging mass spectrometry methods such as desorption electrospray ionization mass spectrometry (DESI) and matrix-assisted laser desorption ionization (MALDI) can probe material at similar length scales (typically $10 \mathrm{~s}$ of

Received: July 5, 2018

Accepted: September 17, 2018

Published: September 27, 2018 
microns), DAPNe offers the possibility of providing more detailed molecular speciation from localized areas. This is first due to the long spray times ( $>20 \mathrm{~min}$ ) that can be achieved via nanospray, with the possibility of performing collision-induced dissociation (CID) transitions to confirm the molecular identification of the analytes. Additionally, the decoupling of sample extraction and analysis in DAPNe offers the possibility for sample manipulation prior to analysis to increase sensitivity and selectivity. ${ }^{2,3}$ For example, it is possible to separate lipophiles from hydrophiles after extraction to reduce matrix effects. ${ }^{2}$ Using LESA, it has been shown that proteins can be captured in a pipet tip and digested prior to analysis, allowing bottom-up identification, ${ }^{12}$ and a single approach could be implemented in the future with DAPNe.

Although DAPNe has been reported to have excellent sensitivity, ${ }^{11}$ the quantitative capabilities of the technique are yet to be fully investigated. For DAPNe to realize its full potential in biosciences, many applications will require quantitative data. For example, quantification of local analyte concentrations in tissue is important in understanding drug delivery and disease progression. ${ }^{13,14}$ This study therefore assesses for the first time the possibility of providing quantitative data from tissues using DAPNe.

For localized quantification of analytes in tissue, a successful outcome generally depends on appropriate deposition of internal standard. Various approaches exist in the imaging mass spectrometry field. ${ }^{15-17}$ These include normalization to a matrix compound that was homogeneously deposited with MALDI $^{13}$ and DESI, ${ }^{18}$ addition of internal standard to the spray solvent with nano-DESI, ${ }^{19}$ and normalizing to the total ion count. ${ }^{20-23}$ Another method that has been used is a tissue extraction coefficient (TEC), to account for various ion suppression factors in a tissue sample. ${ }^{24,25}$ We consider a selection of these approaches here for compatibility with DAPNe. One potential advantage of DAPNe over other techniques is that the diameter of the spot can be measured (by visualizing the wetted area under a microscope directly after extraction), and this allows for another normalization (the spot area) to be used. In this work, we assess the feasibility of normalizing an analyte signal to (a) the sampling area of the extraction spot, (b) selected matrix compounds, (c) isotopically labeled internal standard sprayed on the surface, and (d) an isotopically labeled internal standard in the extraction solvent. Comparing the efficacy of possible normalization methods is also of interest across other surface mass spectrometry techniques.

\section{MATERIALS AND METHODS}

Two standard substrates were used. The first standard substrate was a gelatin film, which was used due to the possibility of producing spatially uniform films spiked with drug compounds, as shown in Figure 2. Along with investigation of different methods of addition of isotopically labeled internal standards, "model samples" could readily be produced from the gelatin films, wherein the films were spiked with both analyte AND internal standard (for colocation). This allowed any uncertainty due to a difference in location of internal standard and analyte to be decoupled from other sources of variation.

The second standard substrate was a set of tissue homogenates. These homogenates have been previously used by Swales et al. as calibration standards in order to quantify local concentrations of drugs using surface mass spectrometry
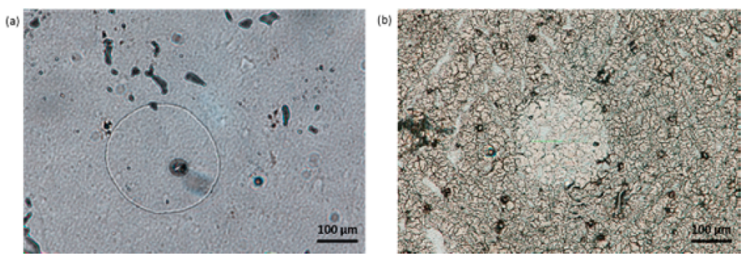

Figure 2. Optical images of (a) gelatin sample and (b) tissue homogenate, where the scale coincides to $100 \mu \mathrm{m}$. The samples were viewed under a Nikon AZ100 microscope, and the diameter of the spot size (solvent footprint) was measured using the NIS-D Elements software.

techniques LESA and DESI. ${ }^{16}$ The use of tissue homogenates provides a sample of a similar matrix to many envisaged applications in biosciences. Figure 2 shows a photograph of both the tissue homogenate and a spiked gel, captured using a Nikon AZ100 microscope.

Gel Sample Preparation. Gelatin powder (Sigma-Aldrich, U.K.) was dissolved in deionized water heated to $60{ }^{\circ} \mathrm{C}$ to make a stock solution of $0.3 \mathrm{~g} / \mathrm{mL}$. An aliquot of gelatin solution was spiked with cocaine $(304.1549 \mathrm{~m} / z)$ and BZE $(290.1392 \mathrm{~m} / z$ ) (Sigma-Aldrich, U.K.) to $1000 \mathrm{ng} / \mathrm{mL}$. A second aliquot of gelatin was spiked with cocaine, BZE, cocaine- $d_{3}$, and BZE- $d_{3}$ to the same concentration. A control (blank) gelatin sample and the spiked samples were dried in a vacuum oven at $14.5 \mathrm{psi}$ for $5 \mathrm{~h}$. Once dried, the gelatin samples were cut and placed on glass slides using double-sided tape.

Tissue Homogenates. Liver mimetics were prepared as described by Swales et al. ${ }^{16}$ Liver tissue was homogenized using a Fisher Powergen 500 homogenizer for a minimum of $30 \mathrm{~s}$. Appropriate volumes containing a solution of olanzapine were spiked into the homogenates to form liver homogenate samples of 10 and $100 \mathrm{nmol} / \mathrm{g}$ (assuming that $1 \mathrm{~mL}$ of solution is equivalent to $1 \mathrm{~g}$ of homogenate). The homogenates were then frozen at $-80{ }^{\circ} \mathrm{C}$ prior to sectioning. The homogenates were sectioned at the National Physical Laboratory (NPL) to $10 \mu \mathrm{m}$ thickness using a Thermo cryosection NX70 (ThermoScientific, U.K.) and thaw mounted onto Super Frost Plus slides (ThermoScientific, U.K.). The prepared slides were then vacuum-packed post sectioning and frozen at -80 ${ }^{\circ} \mathrm{C}$. Prior to analysis, samples were taken out of the freezer and left to reach room temperature.

Internal Standard Addition. An internal standard (IS) solution of cocaine- $d_{3}$ and BZE- $d_{3}(1000 \mathrm{ng} / \mathrm{mL})$ in $100 \%$ acetonitrile (ACN) was prepared (Sigma-Aldrich, U.K.) to be used for the gel samples. An IS solution of olanzapine- $d_{8}(1000$ $\mathrm{ng} / \mathrm{mL}$ ) was prepared for the tissue homogenates. Internal standard was added via three different methods: (a) directly added by electrospray using a TM Sprayer (HTX Imaging, USA), (b) a $5 \mathrm{ng} / \mathrm{mL}$ IS solution in 90:10 ACN $/ \mathrm{H}_{2} \mathrm{O}$ with $0.1 \%$ formic acid (FA) extraction solvent, and (c) in the gelatin solution to represent a model sample. Method (c) could only be applied for the gel samples as the tissue homogenates were received prespiked. Table 1 summarizes the three sample sets prepared. The internal standards were sprayed for 40 passes on the gel samples at a velocity of $1333 \mathrm{~mm} / \mathrm{min}$ at a flow rate of $0.07 \mathrm{~mL} / \mathrm{min}$, with a track spacing of $3 \mathrm{~mm}$, with alternating horizontal and vertical direction, similar to Steven et al. ${ }^{26}$

DAPNe-NSI-MS. Other than when the internal standard ( 5 $\mathrm{ng} / \mathrm{mL}$ ) was incorporated in the extraction solvent, substrates were probed using 90:10 ACN $/ \mathrm{H}_{2} \mathrm{O}$ with $0.1 \%$ FA. The 
Table 1. Summary of Sample Sets and Internal Standard Addition Methods for Gel and Homogenate Samples

$\begin{array}{llc}\text { samples } & \begin{array}{c}\text { internal standard addition } \\ \text { method }\end{array} & \begin{array}{c}\text { internal standard } \\ \text { concentration }(\mathrm{ng} / \mathrm{mL})\end{array} \\ & \begin{array}{c}\text { direct addition via } \\ \text { pneumatic spray } \\ \text { DAPNe extraction solvent } \\ \text { gelatin solution (model } \\ \text { sample) }\end{array} & 1000 \\ & \begin{array}{c}\text { direct addition via } \\ \text { pneumatic spray } \\ \text { DAPNe extraction solvent }\end{array} & 1000 \\ & \end{array}$

solvents were loaded into palladium/gold $(\mathrm{Pd} / \mathrm{Au})$ coated borosilicate capillary tips with an internal diameter of $1 \mu \mathrm{m}$ (MSWil, Switzerland). The capillary tip was guided to the region of interest (ROI) using nanomanipulators (Attocube, Germany). The extraction solvent was injected at a pressure of $5 \mathrm{psi}$ for $0.2 \mathrm{~s}$ and was reaspirated back into the tip for $0.4 \mathrm{~s}$. The injection and reaspiration of the solvent were controlled by a PM1000 microinjector (MicroData Instrument, USA). The injection of extraction solvent leaves a visible footprint on the sample surface, and we define the diameter of this wetted region as the "spot size." A different spot was used for each extraction $(n=5)$, where the average spot size for the gel samples was $340 \mu \mathrm{m}$, ranging from 90 to $500 \mu \mathrm{m}$. The same approach was used for the tissue homogenates, where the average spot size for all extractions was $215 \mu \mathrm{m}$, ranging from 178 to $280 \mu \mathrm{m}$. Samples for extraction were viewed under a Nikon AZ100 microscope, and spot sizes were measured using the NIS-D Elements software (Nikon, U.K.).

After extraction, solvent and extracted analytes were introduced into a Thermo Orbitrap Q-Exactive Plus mass spectrometer (Thermo Scientific, Germany) via a Nanospray Flex Ion source (Thermo, U.K.). A potential of $2 \mathrm{kV}$ was applied to the capillary tip. Full scan data was acquired for $30 \mathrm{~s}$ using a mass range of $50-500 \mathrm{~m} / z$ for the gelatin samples and $100-1200 \mathrm{~m} / \mathrm{z}$ mass range for the tissue homogenate samples at a mass resolving power of 280000 at $200 \mathrm{~m} / z$. The data were acquired with the automatic gain control (AGC) on and set to $1 \mathrm{e}^{6}$ ions. Data for both analytes and internal standard were integrated over the time during which the analytes of interest were detected in the extracted ion chronogram (XIC).

\section{RESULTS AND DISCUSSION}

Nanospray Variability. Investigations into the contribution of the nanospray to the variability of the DAPNe technique were carried out, through direct ionization of standards of cocaine and benzoylecgonine (BZE) at concentration of $5 \mathrm{ng} / \mathrm{mL}$. One of the experimental parameters that was found to affect variability was the distance of the capillary tip to the skimmer cone. Figure 3 shows the coefficient of variation $(\mathrm{CV})$ of $[\mathrm{M}+\mathrm{H}]^{+}$ion signals for cocaine (304.1549 $\mathrm{m} / z)$, BZE $(290.1392 \mathrm{~m} / z)$, and the corresponding internal standards (cocaine- $d_{3}$ and BZE- $d_{3}$ ) at varying distances of the capillary tip to the skimmer cone. Each replicate $(n=5)$ was carried out using a different $\mathrm{Pd} / \mathrm{Au}$ capillary tip. Figure 3 shows that at $25 \mathrm{~mm}$, the variability of cocaine intensity was the lowest, in comparison to the other measured distances. However, at this distance, the cocaine signal was an order of magnitude lower than (e.g.) a distance of $18 \mathrm{~mm}$. The average intensities for both cocaine and BZE were $3 \mathrm{e}^{6}$ and $6 \mathrm{e}^{5}$ respectively at $18 \mathrm{~mm}$, which was the highest intensity in comparison to the other distances (see Supporting Information Figure 1). Therefore, $18 \mathrm{~mm}$ was adopted for sequential measurements due to optimal sensitivity and precision.

The data show that at the selected geometry of $18 \mathrm{~mm}$, the variability in signal between replicate measurements was considerably high, at the $20-40 \%$ level. Normalization of the analyte to the internal standard (IS) to provide a ratio (A/IS) reduced the $\mathrm{CV}$ between replicate measurements to $6 \%$ and $8 \%$ for cocaine and BZE respectively. Therefore, without normalization to an internal standard, the nanospray method itself introduces significant variability. This could be due to differences in the ion droplet formation due to the tip to tip variation. $^{28}$ Corkery et al. reported that in the chip-based
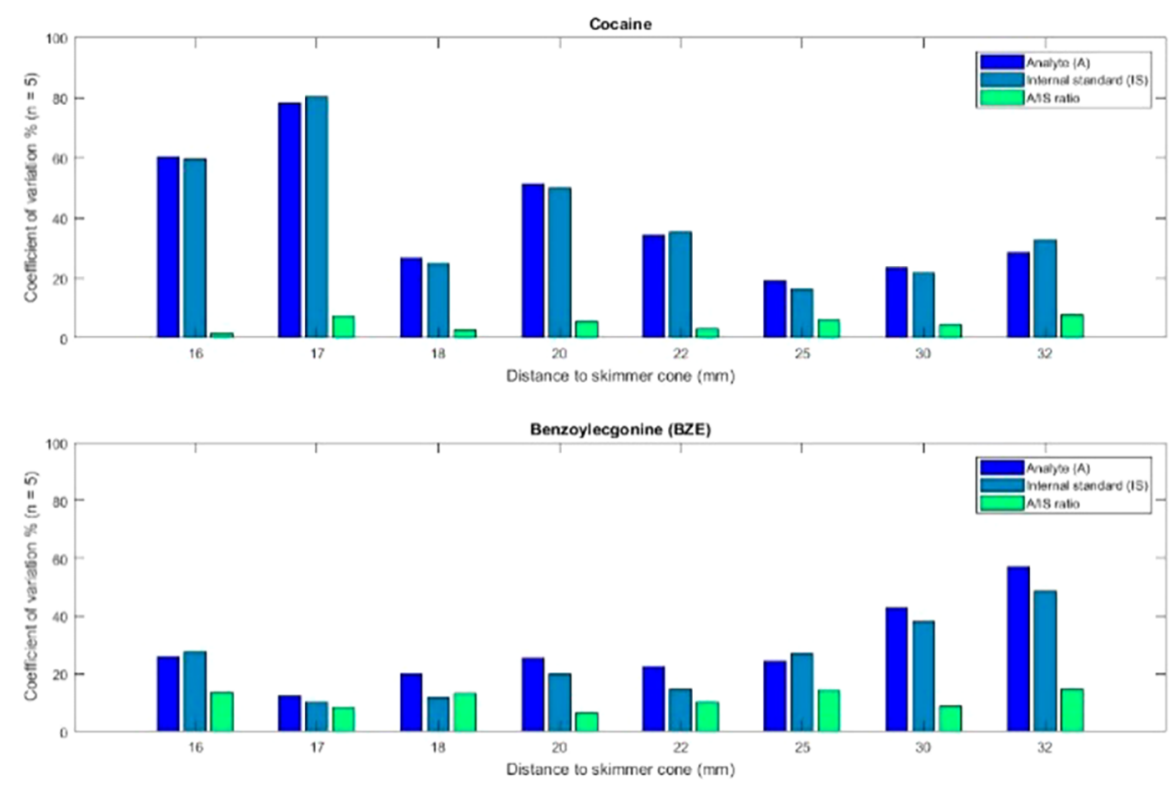

Figure 3. Coefficient of variation $(n=5)$ of cocaine and BZE (analytes) and cocaine- $d_{3}$ and BZE (internal standard) at varying distances of capillary tip to the skimmer cone. 

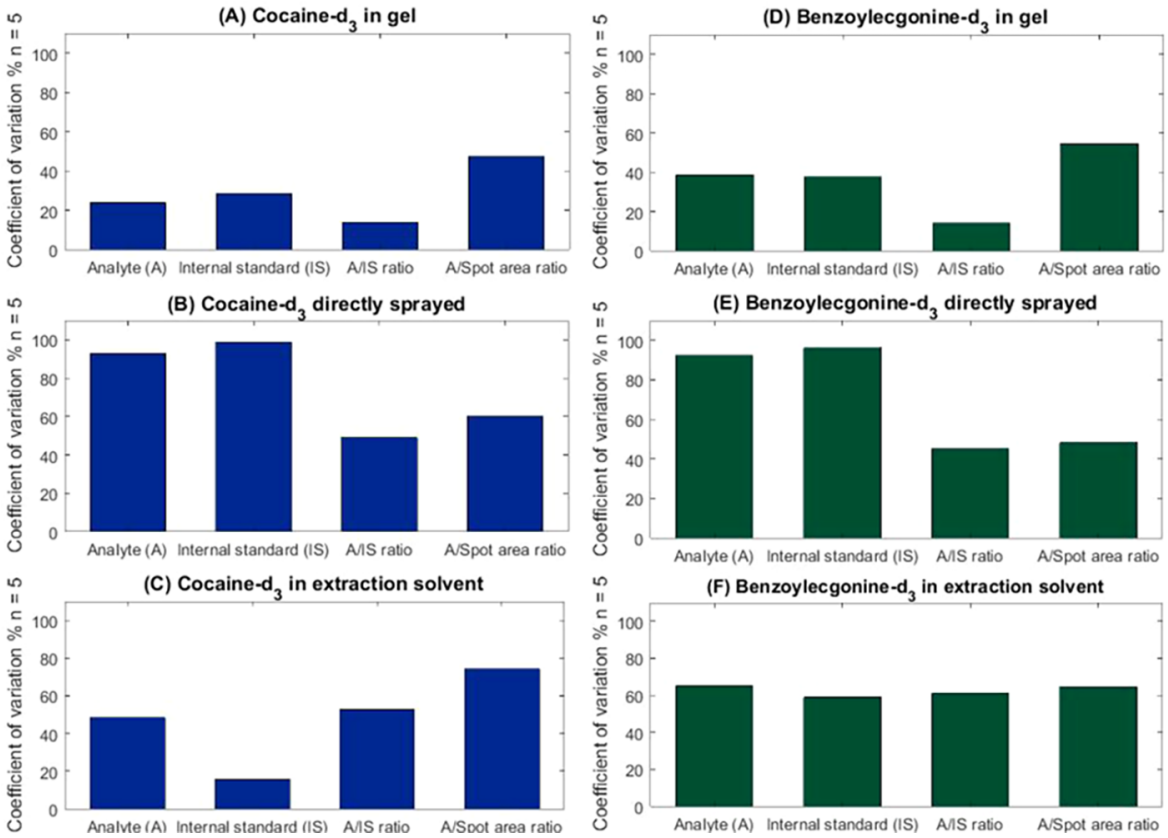

Figure 4. Coefficient of variation $(n=5)$ of cocaine $(a-c)$ and benzoyylecgonine $(d-f)$ analytes with different methods of internal standard addition.
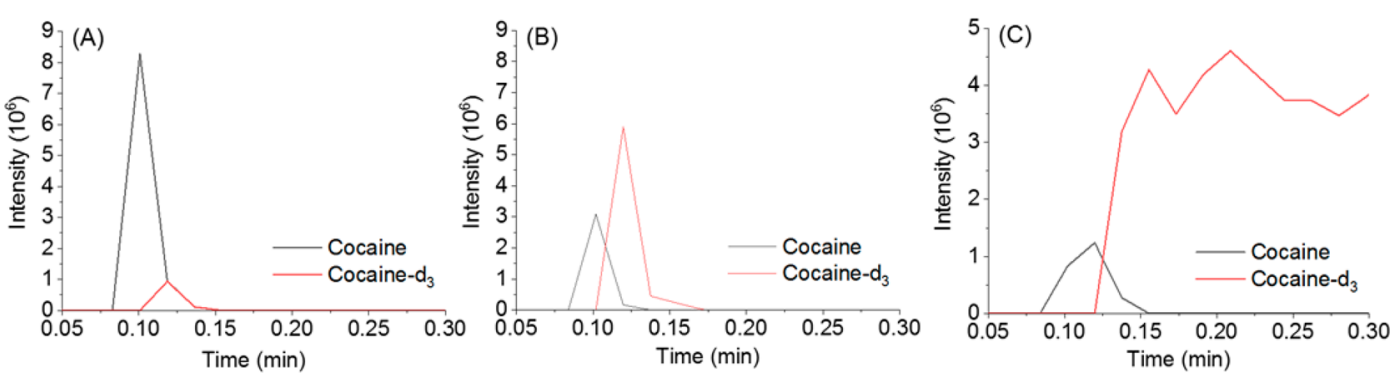

Figure 5. Example extracted ion chronograms of cocaine (black line) and cocaine- $d_{3}$ (red line) for the first extraction of the internal standard (a) in the gel, (b) directly sprayed on the gel, and (c) in the extraction solvent.

nanospray system, the nozzle to nozzle variability of the analyte to internal standard ratio was $5 \%{ }^{27}$ This highlights that normalization is required, even with NSI, in order to reduce the variability.

Internal Standard in the Gelatin Solution (Model Samples). Figure $4 \mathrm{a}$ and $4 \mathrm{~d}$ show the $\mathrm{CV}$ of the raw and normalized data for cocaine and BZE respectively for the model sample, where both analyte and internal standard were located in the gel substrate (see Table 1). Here, the variability for cocaine and BZE without normalization was $20 \%$ and $40 \%$ respectively. When the analytes were normalized to the internal standard, the CV reduced to below $15 \%$ for both analytes. This shows that although the CV is similar compared with direct ionization of standards (presented above), DAPNe can provide a good level of precision, provided that the analytes are colocated with an internal standard. The spot area was also used to normalize the analyte signal but was found not to be as effective in reducing the variability as normalization to the internal standard.

Comparison of Two Other Internal Standard Addition Methods. Addition of internal standard (IS) by spraying was investigated for suitability for practical applications, where the only way to add an internal standard is to add it subsequently to formation of gel samples. Figure $4 \mathrm{~b}$ and $4 \mathrm{e}$ show that the raw signal intensity for cocaine and BZE varied across the five replicates by more than $90 \%$, which reduced to $48 \%$ and $45 \%$ respectively when normalized to the internal standard signal. Normalizing to the spot area also reduced the $\mathrm{CV}$ but was not as effective as normalizing to the internal standard.

A higher throughput method of internal standard incorporation is to locate it in the extraction solvent. The signal intensities of cocaine and BZE after normalization to an internal standard in the extraction solvent are shown in Figure $4 \mathrm{c}$ and $4 \mathrm{f}$ respectively. The CV for cocaine and BZE was $48 \%$ and $65 \%$ respectively without normalization. Normalization of the analyte to either the internal standard or the spot size did not significantly reduce the variability of the data when the internal standard was incorporated in the extraction solvent.

Figure 5 shows extracted ion chronograms (XICs) for cocaine and cocaine- $d_{3}$ for one replicate of each sample preparation method. The XICs for the model sample (Figure 5a) and for the pneumatically sprayed internal standard (Figure $5 b$ ) are broadly similar, with the analyte and internal standard being detected in sequential scans. In contrast, when the internal standard was in the spray (Figure 5c), the XIC for cocaine- $d_{3}$ shows the cocaine from the gel being detected before (approximately 3 scans) the internal standard because 

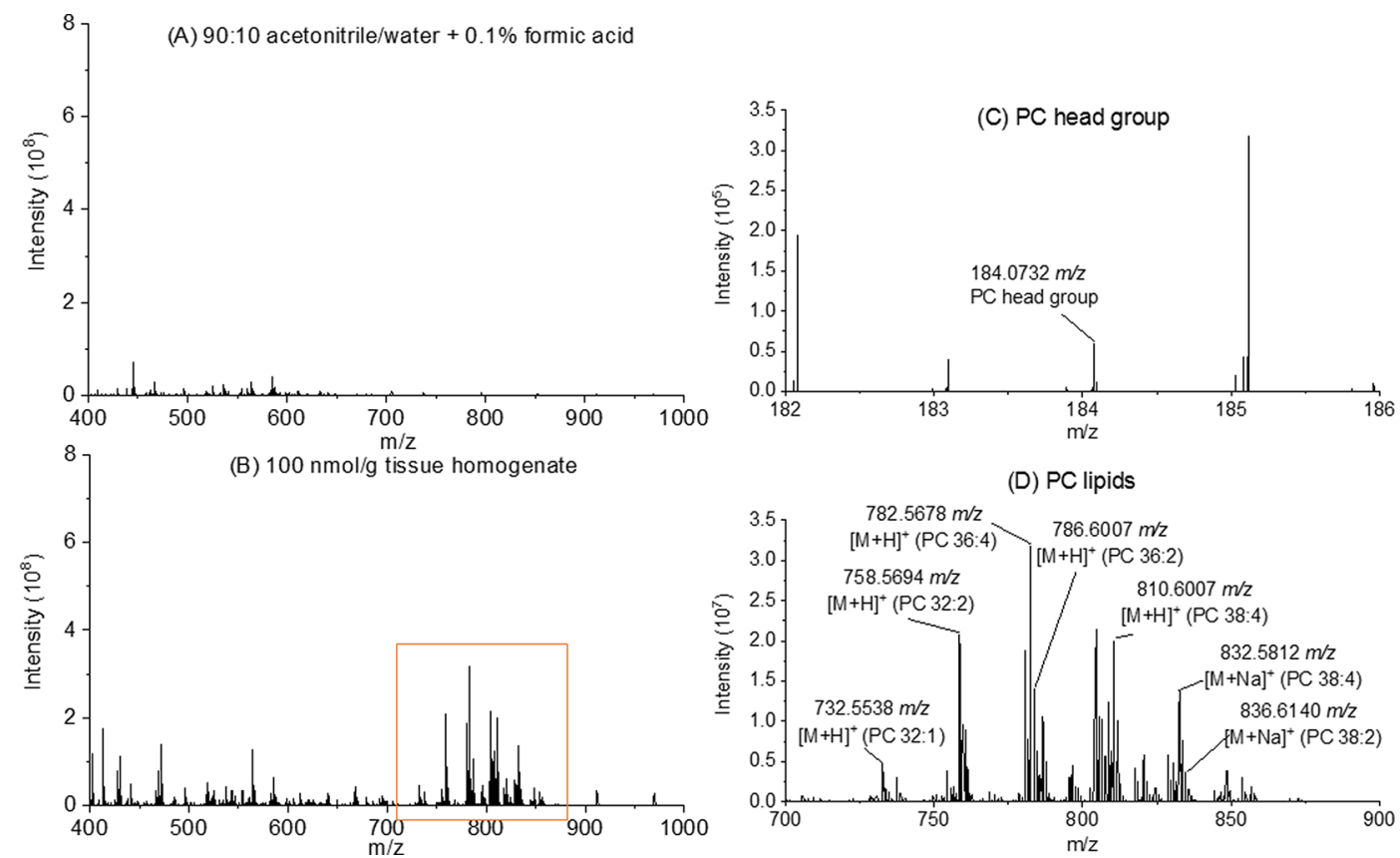

Figure 6. Mass spectrum of (a) 90:10 acetonitrile/water with $0.1 \%$ formic acid extraction solvent and (b) extraction of $100 \mathrm{nmol} / \mathrm{g}$ tissue homogenates, (c) PC headgroup, and (d) PC lipids.
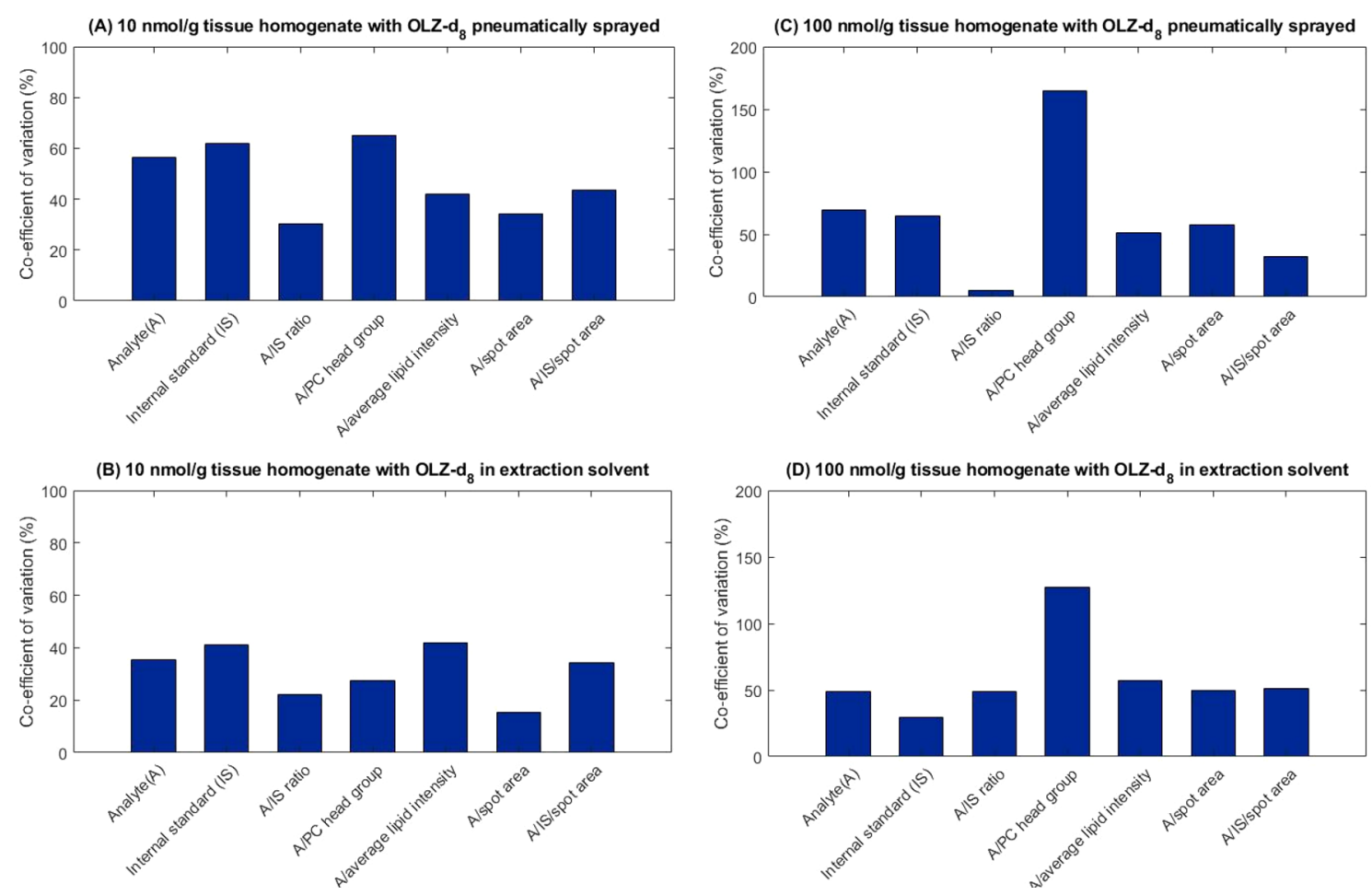

Figure 7. Coefficient of variation for $10 \mathrm{nmol} / \mathrm{g}(\mathrm{a}, \mathrm{b})$ and $100 \mathrm{nmol} / \mathrm{g}(\mathrm{c}, \mathrm{d})$ tissue homogenates where the internal standard was pneumatically sprayed or added in the extraction solvent.

analytes extracted from the sample are front-loaded onto the nanospray emitter tip. This is presumably the reason why normalization to internal standard failed to reduce the variability for this set of samples. It was found that normalizing to the signals detected over the time during which the internal standard was detected (internal standard detection time) led to a high CV (see Supporting Information Figure 2). Therefore, the signals over the time window during which the analyte was detected (analyte detection time) were used for normalization. This results in a lower CV in general; see Supporting Information Figure 2.

We have observed that the front loading effect can be reduced by waiting for a longer period of time for analytes to diffuse into the extraction solvent. However, we do not consider this approach in the present work for the following reasons. First, due to the amount of extracted material being 
different every time, it is difficult to decouple this variable from the wait time. Second, adding a wait time reduces the measurement throughput. In the introduction, we state that an advantage of DAPNe is the long nanospray time that can be achieved, but it should be made clear that there is a trade-off to be made between sensitivity and throughput (achieved by front loading) and long spray times (achieved by waiting for analytes to diffuse into the tip).

Normalization to Matrix Compounds. In certain instances, an isotopically labeled compound is not available. Therefore, other normalization techniques were explored, such as normalizing to the spot size or the spot area. Another method that could be applied is normalization to matrix compounds. However, for this particular sample set, it was difficult to distinguish a suitable matrix compound; therefore, the feasibility of matrix compound normalization was investigated using a more realistic sample set-a tissue matrix.

Application of Normalization Methods Using Tissue Homogenate Samples Spiked with Olanzapine. DAPNe also showed success in extracting phosphatidylcholine (PC) lipids as well as the spiked drugs in the tissue homogenates. Figure 6 shows a comparison spectra of the extraction solvent only (Figure 6a) and an extraction from a tissue homogenate (Figure 6b). One of the detected compounds was a protonated phosphocholine headgroup at $184.0732 \mathrm{~m} / z$ (Figure 6c) and has been reported by Murphy et al. and Walczak et al. ${ }^{29,30}$ As shown in Figure 7, normalizing the olanzapine signal to this compound did not have a significant impact on the CV. For the higher concentration, the $\mathrm{CV}$ increased to well above $100 \%$. Therefore, normalization to this biological compound does not appear to be a suitable normalization approach. As well as a PC headgroup, various PC lipids were also detected. Seven PC lipids were chosen as a test to determine whether or not normalizing to the average signal for these seven lipids would reduce the $\mathrm{CV}$. These lipids (Figure $7 \mathrm{~d}$ ) had observed masses of $732.5538 \mathrm{~m} / z\left(\mathrm{PC}(32: 1),[\mathrm{M}+\mathrm{H}]^{+}\right), 758.5694 \mathrm{~m} / z$ (PC(32:2), $\left.[\mathrm{M}+\mathrm{H}]^{+}\right), 782.5694 \mathrm{~m} / z\left(\mathrm{PC}(36: 4),[\mathrm{M}+\mathrm{H}]^{+}\right)$, $786.6007 \mathrm{~m} / z$ (PC(36:2), $\left.[\mathrm{M}+\mathrm{H}]^{+}\right), 810.6007 \mathrm{~m} / z$ (PC(38:4), $\left.[\mathrm{M}+\mathrm{H}]^{+}\right), 832.5827 \mathrm{~m} / z(\mathrm{PC}(38: 4),[\mathrm{M}+$ $\left.\mathrm{Na}]^{+}\right)$, and $836.6140 \mathrm{~m} / z\left(\mathrm{PC}(38: 2),[\mathrm{M}+\mathrm{Na}]^{+}\right)$. These lipids were checked against the LipidMaps database. ${ }^{31}$ As shown in Figure 7, this method did not significantly reduce the $\mathrm{CV}$. Of course, the validity of normalizing an exogenous drug signal to a lipid signal may not be the best approach for quantitative analysis in any practical application because lipids are unlikely to be uniformly distributed in real tissue samples. Therefore, normalization to an isotopically labeled internal standard may form a more appropriate normalization approach.

Normalization of Olanzapine in Tissue Homogenates. Figure 7 shows the CV of olanzapine (OLZ) signal when the internal standard olanzapine- $d_{8}\left(\mathrm{OLZ}-d_{8}\right)$, was added to the extraction solvent (Figure $7 \mathrm{a}$ ) and when the internal standard was pneumatically sprayed (Figure $7 \mathrm{~b}$ ) for homogenates spiked at $10 \mathrm{nmol} / \mathrm{g}$. The $\mathrm{CV}$ reduced from $35 \%$ to $22 \%$ when OLZ was normalized to OLZ- $d_{8}$ spiked in the extraction solvent. When OLZ- $d_{8}$ was pneumatically sprayed on the tissue homogenates, the CV reduced from $56 \%$ to $30 \%$. This shows that both IS addition methods applied to the homogenates reduced the $\mathrm{CV}$, which was not the case for the gel samples.

Figure $7 \mathrm{c}$ and $7 \mathrm{~d}$ shows the $\mathrm{CV}$ of olanzapine (OLZ) when olanzapine- $d_{8}$ (OLZ- $\left.d_{8}\right)$ was added in the extraction solvent and pneumatically sprayed for the homogenates spiked at a higher concentration of $100 \mathrm{nmol} / \mathrm{g}$. When the internal standard was located in the extraction solvent, the CV for the A/IS slightly increased from $49 \%$ to $55 \%$. When the IS was pneumatically sprayed, the CV decreased from 69\% to 5\%.

In agreement with the data obtained for the gel samples, addition of internal standard to the solvent did not reduce the variability of DAPNe-NSI-MS. For both types of samples, spray deposition of an internal standard had the greatest effect in improving repeatability. The downside of directly adding the internal standard on the sample is that it increases sample preparation time in comparison to having the IS within the extraction solvent. Additionally, it was observed that the average intensity of olanzapine was higher when the internal standard was spray-deposited in comparison to when the internal standard was in the extraction solvent. This was more prominent in the higher concentration homogenate; however, there was also high variability $(50 \%)$ in the olanzapine signal (see Supporting Information Figure 3). In contrast to the gel samples, less of a front-loading effect was observed (see Supporting Information Figure 4), and using the analyte detection time to obtain the internal standard data produced lower CV than when using the internal standard time (see Supporting Information Figure 5).

Normalizing to the spot area had the general effect of reducing the variability in the tissue homogenates, in contrast to what was observed for the gel samples. This could indicate that the analytes were more evenly distributed in the tissue homogenates (where the variability could be reduced by normalizing to the area) than in the gels (where normalizing to the spot area made little difference). For the low concentration tissue homogenate, normalization reduced the CV from $32 \%$ to $17 \%$, and at the higher concentration, normalization did not significantly reduce the CV. As mentioned previously, the spot area was determined by optical microscopy of the wetted area on the sample immediately following extraction. This does not necessarily reflect the actual sampled area of the substrate. For example, the extraction of analytes may not be uniform over the wetted area because it is possible that not all of the solvent injected is reaspirated back into the capillary tip.

Another approach to normalization that was explored was to normalize the analyte to internal standard ratio, followed by normalization to the spot area. The aim was to use this approach to reduce the variability of measurements taken with the internal standard deposited in the extraction solvent. Normalization to the spot area should correct for any variability in the extracted region, and then normalization to the internal standard would serve the purpose of accounting for variability in the nanospray. However, Figure 7 shows that this normalization approach does not improve measurement precision over normalizing to internal standard only.

The data presented here have only considered one analyte concentration for the gel samples and two analyte concentrations for the tissues. It important to note that many of the normalization problems in direct ionization techniques are due to matrix or saturation effects, so future work is required to test whether the conclusions still hold in the presence of different concentrations of analyte.

\section{CONCLUSIONS}

This study is the first to fully investigate various normalization techniques that can be applied to reduce the variability with DAPNe-NSI-MS. The variability of analyte signal seen during nanospray ionization (reaching more than $60 \%$ in some cases) 
appears to be the dominant source of variability but can be reduced to below $10 \%$ by the use of an isotopically labeled standard. Colocalization of an internal standard with an analyte in a sample allows a precision of below $20 \%$ to be achieved using DAPNe. For practical applications, for example, measurement of local analyte concentration in tissue, the best precision is limited by the ability to colocate analytes and internal standard. The best method of addition of internal standard is by spray deposition, and this generally results in a precision below $30 \%$. Future work will look at improving the repeatability of the nanospray process so that quantitative measurements can be undertaken when an isotopically labeled compound is unavailable. This study has however shown that for DAPNe to be used as a quantitative tool, normalization to an isotopically labeled internal standard, or to the spot size, would be a viable method.

\section{ASSOCIATED CONTENT}

\section{S Supporting Information}

The Supporting Information is available free of charge on the ACS Publications website at DOI: 10.1021/acs.analchem.8b03016.

Average intensities of cocaine, cocaine- $d_{3}$, benzoylecgonine, and benzoylecgonine- $d_{3}$ at carious skimmer distances; comparison of $\mathrm{CV}$ for gel samples and tissue homogenates at different detection windows; comparison of average intensities of olanzapine using two methods of internal standard addition methods and extracted ion chronograms for olanzapine and olanzapine- $d_{8}$, when the internal standard was added using two methods (PDF)

\section{AUTHOR INFORMATION}

\section{Corresponding Authors}

*E-mail: j.dejesus@surrey.ac.uk.

*E-mail: m.bailey@surrey.ac.uk.

ORCID $\odot$

Janella de Jesus: 0000-0002-5075-0112

Notes

The authors declare no competing financial interest.

\section{ACKNOWLEDGMENTS}

This study was possible due to an EPSRC strategic equipment award (EP/P001440/1), an EPSRC sponsored fellowship, EP/ R031118/1, as well as the National Physical Laboratory (NPL)-Surrey studentship fund. Acknowledgments go to Dr. Adam Taylor for helping with sectioning the homogenates at NPL.

\section{REFERENCES}

(1) Horn, P. J.; Ledbetter, N. R.; James, C. N.; Hoffman, W. D.; Case, C. R.; Verbeck, G. F.; Chapman, K. D. J. Biol. Chem. 2011, 286, 3298-3306.

(2) Hamilton, J. S.; Aguilar, R.; Petros, R. A.; Verbeck, G. F. J. Am. Soc. Mass Spectrom. 2017, 28 (5), 918-928.

(3) Phelps, M. S.; Sturtevant, D.; Chapman, K. D.; Verbeck, G. F. J. Am. Soc. Mass Spectrom. 2016, 27 (2), 187-193.

(4) Huynh, V.; Joshi, U.; Leveille, J. M.; Golden, T. D.; Verbeck, G. F. Forensic Sci. Int. 2014, 242, 150-156.

(5) Huynh, V.; Williams, K. C.; Golden, T. D.; Verbeck, G. F. Analyst 2015, 140, 6553-6562.

(6) Phelps, M.; Hamilton, J.; Verbeck, G. F. Rev. Sci. Instrum. 2014, 85,124101 .
(7) Clemons, K.; Wiley, R.; Waverka, K.; Fox, J.; Dziekonski, E.; Verbeck, G. F. J. Forensic Sci. 2013, 58, 875-880.

(8) Schultz, G. A.; Corso, T. N.; Prosser, S. J.; Zhang, S. Anal. Chem. 2000, 72, 4058-4063.

(9) Bailey, M. J.; Randall, E. C.; Costa, C.; Salter, T. L.; Race, A. M.; de Puit, M.; Koeberg, M.; Baumert, M.; Bunch, J. Anal. Methods 2016, 8, 3373-3382.

(10) Randall, E. C.; Bunch, J.; Cooper, H. J. Anal. Chem. 2014, 86, 10504-10510.

(11) Clemons, K.; Dake, J.; Sisco, E.; Verbeck, G. F. Forensic Sci. Int. 2013, 231, 98-101.

(12) Sarsby, J.; Martin, N. J.; Lalor, P. F.; Bunch, J.; Cooper, H. J. J. Am. Soc. Mass Spectrom. 2014, 25, 1953-1961.

(13) Takai, N.; Tanaka, Y.; Inazawa, K.; Saji, H. Rapid Commun. Mass Spectrom. 2012, 26, 1549-1556.

(14) Prideaux, B.; Stoeckli, M. J. Proteomics 2012, 75, 4999-5013.

(15) Hansen, H. T.; Janfelt, C. Anal. Chem. 2016, 88, 11513-11520.

(16) Swales, J. G.; Strittmatter, N.; Tucker, J. W.; Clench, M. R.; Webborn, P. J. H.; Goodwin, R. J. A. Sci. Rep. 2016, 6 (37648), 1-9.

(17) Lanekoff, I.; Thomas, M.; Carson, J. P.; Smith, J. N.; Timchalk, C.; Laskin, J. Anal. Chem. 2013, 85, 882-889.

(18) Hansen, H. T.; Janfelt, C. Anal. Chem. 2016, 88, 11513-11520.

(19) Bergman, H.-M.; Lundin, E.; Andersson, M.; Lanekoff, I. Analyst 2016, 141, 3686-3695.

(20) Bemis, K. D.; Eberlin, L.; Ferreira, C.; Cooks, R. G.; Vitek, O. BMC Bioinf. 2012, 13, A8.

(21) Abbassi-Ghadi, N.; Jones, E. A.; Veselkov, K. A.; Huang, J.; Kumar, S.; Strittmatter, N.; Golf, O.; Kudo, H.; Goldin, R. D.; Hanna, G. B.; et al. Anal. Methods 2015, 7, 71-80.

(22) Tillner, J.; Wu, V.; Jones, E. A.; Pringle, S. D.; Karancsi, T.; Dannhorn, A.; Veselkov, K.; Mckenzie, J. S.; Takats, Z. J. Am. Soc. Mass Spectrom. 2017, 28, 2090-2098.

(23) Randall, E. C.; Race, A. M.; Cooper, H. J.; Bunch, J. Anal. Chem. 2016, 88, 8433-8440.

(24) Hamm, G.; Bonnel, D.; Legouffe, R.; Pamelard, F.; Delbos, J.M.; Bouzom, F.; Stauber, J. J. Proteomics 2012, 75, 4952-4961.

(25) Taylor, A. J.; Dexter, A.; Bunch, J. Anal. Chem. 2018, 90, 56375645.

(26) Steven, R. T.; Dexter, A.; Bunch, J. Methods 2016, 104, 111117.

(27) Corkery, L. J.; Pang, H.; Schneider, B. B.; Covey, T. R.; Siu, K. W. M. J. Am. Soc. Mass Spectrom. 2005, 16, 363-369.

(28) El-Faramawy, A.; Siu, K. W. M.; Thomson, B. A. J. Am. Soc. Mass Spectrom. 2005, 16, 1702-1707.

(29) Murphy, R. C.; Axelsen, P. H. Mass Spectrom. Rev. 2011, 30 (4), 579-599.

(30) Walczak, J.; Bocian, S.; Buszewski, B. Food Anal. Methods 2015, 8 (3), 661-667.

(31) Gateway, L. Lipid Maps Structure Database; http://www. lipidmaps.org/data/LMSDRecord.php?\&LMID=LMGP01010788 (accessed Jul 4, 2017). 